\title{
Geschlechtsspezifische Lohnungleichheit in Betrieben, Berufen und Jobzellen (1993-2006)
}

\author{
Hermann Gartner · Thomas Hinz
}

\begin{abstract}
Zusammenfassung: Während die formalen Ungleichheiten zwischen Frauen und Männern bei Bildungsabschlüssen und die Unterschiede im Erwerbsverhalten im Zeitverlauf in Deutschland zurückgehen, präsentiert sich der geschlechtsspezifische Lohnunterschied unverändert stabil. Dies ist insbesondere deshalb erstaunlich, weil der Lohnunterschied verstärkt in den Blick der Antidiskriminierungsdiskussion geraten ist. Unter Verwendung von Daten des Instituts für Arbeitsmarktund Berufsforschung (IAB) untersucht der Beitrag, ob die durchschnittlichen Lohnunterschiede zwischen vollzeitbeschäftigten Frauen und Männern mit der unterschiedlichen Verteilung der Geschlechter auf Wirtschaftsbereiche, Berufe und Betriebe erklärt werden können. Als kleinste Analyseebene werden gleiche Berufe innerhalb von Betrieben betrachtet (Jobzellen). Damit wird für den Analysezeitraum von 1993 bis 2006 eine bestmögliche Annäherung an den „within-job wage gap" erzielt. Es zeigt sich: Bei gleicher Ausbildung und gleicher Berufserfahrung verdienen Frauen in solchen Jobzellen 12\% weniger als Männer. In zeitlicher Hinsicht hat sich der Lohnabstand trotz Veränderungen in der Bildungsbeteiligung, der Zusammensetzung der Erwerbstätigen und des politischen Gleichstellungsdrucks nicht verändert. Am Ende des Beitrags diskutieren wir inhaltliche und methodische Gründe, die für dieses empirische Bild verantwortlich sein könnten.
\end{abstract}

Schlüsselwörter: Diskriminierung · Direkte Diskriminierung · Evaluative Diskriminierung · Geschlechtsspezifischer Lohnunterschied · Lohnungleichheit

\section{Gender wage inequality in firms, occupations, and job-cells (1993-2006)}

\begin{abstract}
Whereas educational inequalities between women and men and differences in labor market participation shrinked or even diminished during the last decades, the gender pay gap remained stable over time. This is remarkable because the pay gap has attracted much more attention as the main target of anti-discrimination policies. Using data from the IAB (Institute for Employment Research), we analyze whether the average pay gap between women and men working full-time can be explained by their employment in different industries, occupations, and firms. As the smallest level of analysis we focus on occupations within firms (job cells). This strategy
\end{abstract}

H. Gartner (ه)

Institut für Arbeitsmarkt- und Berufsforschung (IAB)

Regensburger Straße 104, 90478 Nürnberg, Deutschland

E-Mail: hermann.gartner@iab.de

T. Hinz, Konstanz, Deutschland 
of analysis yields the best possible approximation to the concept of "within-job wage gap". The results show that women with equivalent training and occupational experience earn wages that are 12 percent less than the wages of men in such job cells. Even though the educational participation of women rose to that of their male counterpart, the gender composition of labor market participation changed and the pressure of equal employment policies grew, the gender wage gap does not decrease within our observation period (1993-2006). In conclusion, we discuss theoretical and methodological reasons for this finding.

Keywords: Discrimination - Direct discrimination · Evaluative discrimination - Wage inequality $\cdot$ Gender pay gap

\section{Les inégalités de salaire entre hommes et femmes dans les entreprises, les métiers et les cellules d'emploi (1993-2006)}

Résumé: Tandis que les inégalités formelles entre hommes et femmes en matière de diplômes et les différences en termes de parcours professionnel diminuent avec le temps en Allemagne, les écarts de salaire entre hommes et femmes restent stables. Ce fait est d'autant plus étonnant que les écarts de salaire ont fait l'objet d'une plus grande attention dans le cadre du débat sur la lutte contre la discrimination. Cet article étudie à partir de données de l'Institut für Arbeitsmarkt- und Berufsforschung (Institut de recherche sur le marché de l'emploi et les professions) si les écarts de salaire moyens entre hommes et femmes employés à plein temps peuvent s'expliquer par l'inégale répartition des hommes et des femmes entre différents secteurs économiques, professions et entreprises. Les personnes exerçant la même profession au sein d'une même entreprise (cellules d'emploi) ont été retenues comme niveau d'analyse le plus fin. Cette approche permet d'appréhender avec la plus grande précision possible les écarts de salaire au sein d'une même profession (,within-job wage gap") sur la période analysée de 1993 à 2006. Il apparaît ainsi que dans de telles cellules d'emploi, les femmes gagnent 12 pourcent de moins que les hommes à niveau d'éducation et expérience professionnelle égale. Les écarts de salaire n'ont pas évolué dans le temps en dépit de changements dans l'accès à l'éducation et la composition de la population active et de la pression politique en faveur de la lutte contre la discrimination. À la fin de cet article, nous discutons les raisons de fond et de méthode qui pourraient rendre compte de ce tableau empirique.

Mots-clés: Discrimination · Discrimination directe · Discrimination évaluative · Écarts de salaire entre hommes et femmes · Inégalités de salaire

\section{Einleitung}

Seit 2008 wird in Deutschland mit Unterstützung der Bundesregierung der equal pay day begangen. Das genaue Datum des equal pay day variiert dabei von Jahr zu Jahr und von Land zu Land (2009 war es in Deutschland der 20. März) und steht für den Zeitraum, den Frauen in Deutschland seit Jahresbeginn länger arbeiten müssten, um den gleichen Lohn zu erhalten wie Männer im vergangenen Jahr. Diese öffentlichkeitswirksame Thematisierung des geschlechtsspezifischen Lohnunterschieds zeigt, wie stark der öffentliche und politische Druck inzwischen geworden ist, wenn die Diskussion auf, ,gleiche Löhne“ für gleiche oder gleichwertige Arbeit kommt. Die Lohnlücke beläuft sich aktuell laut der Berechnungsmethode der Europäischen Union auf 23\% und ist 2008 sogar größer aus- 
gefallen als in den Vorjahren (Kommission der europäischen Gemeinschaften 2009). Dieser geschlechtsspezifische Lohnunterschied gilt in Deutschland wie in anderen modernen Erwerbsgesellschaften zunehmend als illegitim, weil sich die Unterschiede in den Ausbildungsabschlüssen zwischen Männern und Frauen in epochaler Weise verringert haben. Im Hinblick auf die formalen Abschlüsse der Schul- und Universitätsabgänger haben Frauen bekanntlich gleichgezogen bzw. die Männer sogar überholt. Die ungleichen Löhne werden verstärkt als problematisch wahrgenommen, da die Rendite für ,Investitionen“ von Frauen in Humankapital scheinbar geringer ausfällt und dies auch gesamtwirtschaftliche Nachteile mit sich bringt.

Wie die Lohnlücke zwischen den Geschlechtern zu erklären ist, ist Gegenstand unzähliger theoretischer Abhandlungen (Überblick bei Roos u. Gatta 1999; Blau et al. 2006) und empirischer Forschungen, bei denen unterschiedliche Arten von Diskriminierung hervorgehoben werden (zur Diskriminierung allgemein: Lorenz 1993; Darity u. Mason 1998; Altonji u. Blank 1999; Pager u. Shephard 2008). Unterschieden werden dabei unmittelbare und mittelbare Diskriminierung, allokative und evaluative Diskriminierung sowie statistische und präferenzbasierte Diskriminierung. Unmittelbare Diskriminierung liegt im rechtlichen Sinn vor, wenn eine Person wegen ihres Geschlechts oder anderer nicht produktivitätsbezogener Faktoren in einer vergleichbaren Situation eine weniger günstige Behandlung erfährt als eine andere Person (EU Richtlinie 2000/78/Art. 2,1). Mittelbare Diskriminierung liegt vor, wenn die nicht gerechtfertigte Ungleichbehandlung durch dem Anschein nach neutrale Vorschriften oder Verfahren begründet wird. Allokative Diskriminierung bezieht sich auf die Platzierung für unterschiedliche Aufgaben und Positionen bei gleichen Voraussetzungen. Evaluative Diskriminierung ist die ungleiche Bewertung gleicher Leistungen. Am deutlichsten wird gegen das Diskriminierungsverbot verstoßen, wenn für die gleiche berufliche Tätigkeit, bei gleicher Arbeitszeit im gleichen Unternehmen (in sogenannten Jobzellen) unterschiedliche Löhne gezahlt werden. Diese direkte Form der Lohndiskriminierung ist allerdings bislang in Deutschland selten untersucht worden, weil die entsprechenden Datengrundlagen erst seit kurzer Zeit zur Verfügung stehen. Hierzu werden betriebsbezogene Lohninformationen benötigt, die in vielen Standarderhebungen der Sozialforschung fehlen. In Ergänzung einer eigenen Untersuchung (Hinz u. Gartner 2005) soll zunächst die Größenordnung der berufs- und betriebsbezogenen Lohnlücke für den Zeitraum 1993-2006 möglichst präzise bestimmt werden. Wir betrachten dabei nur Vollzeitbeschäftigte in gleichen Berufen und gleichen Betrieben, um uns optimal der Frage nach dem Ausmaß der unmittelbaren, evaluativen Diskriminierung (und damit einem zentralen Teil der illegitimen Lohnunterschiede) anzunähern. Andere Aspekte - wie die Frage der beruflichen Segregation, der Spaltung des Arbeitsmarkts in Segmente oder nach Arbeitszeit - werden zugunsten einer größeren analytischen Schärfe ausgeblendet. Wir verwenden ein einfaches, aber aussagekräftiges multivariates Analyseverfahren, nämlich Lohnschätzungen mit fixen Effekten, welches über die Lohnunterschiede zwischen Männern und Frauen informiert, die auf der gleichen Analyseebene, also etwa im gleichen Beruf, im gleichen Wirtschaftsbereich oder in der gleichen Jobzelle tätig sind.

Der vorliegende Beitrag ergänzt die Diskussion zu illegitimen Lohnunterschieden in dreierlei Hinsicht: Wir untersuchen, ob sich die Lohnlücke schließt, wenn neben der Ausbildung auch die tatsächliche Berufserfahrung der Frauen und Männer berücksichtigt wird. Wir fragen danach, wie sich der „,within-job wage gap“ angesichts einer stär- 
keren Orientierung an expliziten Verboten von direkter und indirekter Diskriminierung entwickelt hat; im Jahr 2000 hat die EU eine entsprechende Richtlinie vorgelegt, die in Deutschland in das allgemeine Gleichbehandlungsgesetz (AGG) von 2006 mündete. Schließlich stellen wir dar, inwieweit sich hinter der Persistenz der durchschnittlichen Lohnlücke Veränderungen der geschlechtsspezifischen Lohnverteilungen verbergen.

\section{Lohnungleichheiten: Nachweise oder nur Indizien für Diskriminierung?}

Nach allgemeiner Auffassung entstehen illegitime Lohnunterschiede, wenn auch bei gleicher Arbeitszeit, Bildung, Ausbildung und Tätigkeit eine Lohnlücke zwischen Frauen und Männern besteht. Dabei spielen die Betriebe als Nachfrager von Arbeitskraft eine Schlüsselrolle. Entweder diskriminieren sie aufgrund von Präferenzen für bestimmte Mitarbeiter (taste for discrimination nach Becker 1971) oder aufgrund von Informationsdefiziten (Arrow 1974; Phelps 1972). Manche produktivitätsrelevanten Eigenschaften von Arbeitnehmern sind vor der Einstellung in den Betrieb im Einzelfall unbekannt, etwa die Einsatzbereitschaft. Wegen solcher Informationsdefizite über die tatsächliche Produktivität von Mitarbeitern werden diese von Arbeitgebern so behandelt, als würden sie dem Durchschnitt ihrer sozialen Gruppe entsprechen. Weil Arbeitgeber davon ausgehen, dass Frauen im Durchschnitt häufiger ihre Erwerbstätigkeit unterbrechen als Männer und solche Erwerbsunterbrechungen mit Produktivitätseinbußen verbunden sind, stellt ein Arbeitgeber eine hoch motivierte und karrierebereite Bewerberin möglicherweise auf eine weniger entwicklungsfähige Position ein oder zahlt ihr einen geringeren Lohn.

Die empirische Arbeitsmarktforschung hat bis in jüngste Zeit belegt, dass trotz gleicher Arbeitszeit, Bildung, Ausbildung und Berufserfahrung ungleicher Lohn gezahlt wird (Blau u. Kahn 1996; für Deutschland beispielsweise: Diekmann et al. 1993; Fitzenberger et al. 2004). Dieser Stand der Forschung beruht überwiegend auf Analysen ohne Berücksichtigung der betrieblichen Ebene (Ausnahmen: Achatz et al. 2005; Heinze u. Wolf 2006; Wolf u. Heinze 2007). Weil darüber hinaus auch weitere, unbeobachtete (nicht gemessene) Eigenschaften wie Einsatzbereitschaft und Unternehmensbindung produktivitätsrelevant sein können, ist umstritten, ob man diese Resultate der Forschung tatsächlich als Beleg für praktizierte Diskriminierung werten muss (etwa Heckman 1998). Diese eher kritische Haltung wird auch dadurch gestützt, dass bei hinreichend genauer Messung der produktivitätsrelevanten Faktoren der empirisch festzustellende Lohnunterschied sehr viel kleiner ausfällt (Lazear 1995; Tam 1997).

Klar ist somit, dass die Frage nach illegitimen Lohnunterschieden auf der Grundlage von Studien, die den Arbeitsmarkt ohne Differenzierung von Branchen und Betrieben untersuchen, nicht zu beantworten ist. Der betriebliche Kontext beeinflusst Prozesse der Lohnsetzung in deutlicher Weise (le Grand et al. 1995; Wolf u. Heinze 2007). ${ }^{1}$ Frauen

1 Betriebe verwenden in der Personalpraxis, beispielsweise bei Beurteilungsgesprächen oder Zielvereinbarungen, geschlechtsspezifisch wirksame Instrumente. So wird in Positionen, bei denen längere Abwesenheiten vom Wohnort nötig sind, auf der Grundlage von Stereotypen ein männliches Profil konstruiert. Weiterhin sind Betriebe relevante soziale Kontexte, wenn es um die Legitimation von Ungleichbehandlung geht (Baron u. Newman 1990; Nelson u. Bridges 1999). 
und Männer werden auf verschiedene Positionen eingestellt, und ihre internen Karriereoptionen sind unterschiedlich (Lazear u. Rosen 1990). Männer gelten als karrierebewusster, und sie verhandeln härter um ihr Gehalt als Frauen (Rupert u. Voigt 2009). Darüber hinaus wirken sich Strukturen und Strategien der Betriebe auf die geschlechtsspezifische Chancenstruktur aus, etwa inwieweit Betriebe die Rückkehr von Müttern nach einer Kinderpause in den Beruf zulassen (vgl. Achatz et al. 2002; Wharton 2002). Erwerbsunterbrechungen sind für die bestehenden Lohnungleichheiten ein wichtiger Faktor (Beblo u. Wolf 2003).

Wenn gleich produktive Personen wegen ihres Geschlechts unterschiedlich in Berufe und Positionen sortiert werden, drückt sich darin eine allokative Diskriminierung aus (Lazear 1995). Allokative Diskriminierung zeigt sich etwa, wenn bei längerer Betriebszugehörigkeit sich aus unterschiedlichen Karriereaspirationen und den damit zusammenhängenden Erwerbsunterbrechungen unterschiedliche Beförderungsraten ergeben. Ein Lohnunterschied zwischen Frauen und Männern, die tatsächlich die gleiche Position und gleiche Berufserfahrung besitzen, also eine direkte Lohndiskriminierung, wäre dann nicht gegeben. Umgekehrt argumentiert: Ein Lohnunterschied im gleichen Beruf und Betrieb gilt am ehesten als ein geeigneter empirischer Nachweis für eine direkte oder evaluative Lohndiskriminierung, weil damit Unterschiede, die sich durch eine unterschiedliche Berufswahl und durch eine Verteilung von Frauen und Männern auf Wirtschaftsbereiche und Betriebe ergeben, ausgeblendet werden. In den USA besteht nur ein sehr geringer „within-job wage gap“ (Petersen u. Morgan 1995; Petersen et al. 1997; Petersen et al. 2003; Petersen u. Saporta 2004). Dort kann der Lohnunterschied nicht mittels direkter Diskriminierung erklärt werden. Es sind offensichtlich angebotsseitige Faktoren wie die Entscheidung für Berufe und Arbeitszeiten sowie nachfrageseitige Faktoren wie die unterschiedliche Platzierung und berufliche Weiterentwicklung von Frauen und Männern, die zu unterschiedlichen Löhnen führen.

Für den deutschen Arbeitsmarkt gab es bis zur Studie von Hinz u. Gartner (2005) keine vergleichbare Untersuchung. Damals konnte erstmals auf der Basis eines für sozialversicherungspflichtig Beschäftigte repräsentativen Employer-Employee-Datensatzes (LIAB) des Instituts für Arbeitsmarkt- und Berufsforschung (IAB) der „,within-job wage gap“ für Westdeutschland ermittelt werden. Die Lohnlücke bei Frauen und Männern mit gleicher Qualifikation in Jobzellen betrug 2001 zwölf Prozent. Dieses Resultat wurde von uns intensiv methodisch diskutiert, wobei vor allem Messprobleme eine zentrale Rolle spielten. Wir kamen zu dem Schluss, dass bei besserer Messung und konservativer Schätzung die tatsächliche Lohnlücke zwischen fünf und sieben Prozent liegen dürfte. Ein wesentlicher Grund für die vermutete Differenz zwischen den Ergebnissen der Regressionsschätzungen und der tatsächlichen Lohnlücke war nach unserer Argumentation die Verwendung der nur grob angenäherten Berufserfahrung, die für die Frauen zu einer Überschätzung der tatsächlichen Berufserfahrung führen musste. Bei entsprechend genauerer Messung sollte der noch verbleibende Lohnunterschied kleiner sein. Im vorliegenden Beitrag können wir dieses Problem aufgrund einer inzwischen verbesserten Datenlage adressieren. Es ist möglich, aus der gesamten Historie sozialversicherungspflichtiger Beschäftigung die tatsächliche Berufserfahrung in Jahren für jeden Beschäftigten im LIAB festzumachen. Wenn - wie in den theoretischen Modellen zum Zusammenhang von Humankapital und Produktivität angenommen - Berufserfahrung eine wesentliche Komponente 
des Humankapitals darstellt, lässt sich unsere erste Hypothese wie folgt formulieren: Die geschlechtsspezifische Lohnlücke in den Jobzellen wird bei genauerer Messung der Berufserfahrung kleiner (H 1).

Ebenso interessant sind die Veränderungen der Lohnlücke im Zeitverlauf. Die Untersuchung von Hinz u. Gartner (2005) war auf die Jahre 1993-2001 beschränkt. Mittlerweile sind Daten bis 2006 verfügbar. Dies erlaubt die Untersuchung einer längeren Zeitreihe, vor allem nach der Verabschiedung der EU-Richtlinie 2000/78, die eine Umsetzung in nationales Recht erforderlich machte. Mit dieser EU-Richtlinie ist ein weit über die Diskriminierung nach dem Geschlecht hinausreichendes Diskriminierungsverbot in Kraft gesetzt worden, das auch im privaten Rechtsverkehr, etwa im Arbeits- oder Wohnungsmarkt, Gültigkeit beansprucht. Die öffentliche Diskussion zur Umsetzung in nationales Recht hielt in Deutschland lange an, sodass erst 2006 das Allgemeine Gleichstellungsgesetz (AGG) verabschiedet wurde. Die Lohnungleichheit zwischen Frauen und Männern war in den Diskussionen ein besonders intensiv beachteter Fall, auch weil bereits vor der EU-Richtlinie 2000/78 Verbote der Lohndiskriminierung im geltenden Recht verankert waren. In den 1990er Jahren wurde das BGB entsprechend geändert (BGB § 611a und § 612, 3): „Bei einem Arbeitsverhältnis darf für gleiche oder für gleichwertige Arbeit nicht wegen des Geschlechts des Arbeitnehmers eine geringere Vergütung vereinbart werden als bei einem anderen Arbeitnehmer des anderen Geschlechts." Bereits in den Römischen Verträgen zur Gründung der Europäischen Gemeinschaft vom 25. März 1957 hieß es in Art. 119: „Jeder Mitgliedstaat wird während der ersten Stufe den Grundsatz des gleichen Entgelts für Männer und Frauen bei gleicher Arbeit anwenden und in der Folge beibehalten." Die EU-Richtlinie von 2000 und die nachfolgende intensive Beschäftigung mit der Lohnungleichheit auf europäischer Ebene (Kommission der europäischen Gemeinschaften 2007) hat die Diskussion in Deutschland um illegitime Lohnungleichheiten erheblich befördert und den allgemeinen Gleichstellungsdruck erhöht (Berkovitch 2001; Ramirez 2001). Es wäre somit zu erwarten, dass die Lohnlücke in den Jobzellen, die am besten dem Konzept der unmittelbaren Diskriminierung entspricht, seit 2000 kleiner wird, weil die Arbeitgeber auf die erhöhten normativen Anforderungen reagieren. Auch wenn dies nicht bedeuten muss, dass die Lohnlücke insgesamt kleiner wird, erwarten wir also einen Rückgang des „within-job wage gap“ (H 2).

\section{Datengrundlage und Analysestrategie}

Im Wesentlichen replizieren wir nachfolgend die Analysen in Hinz u. Gartner (2005) unter Verwendung einer genaueren Messung der Berufserfahrung und einer längeren Zeitreihe (1993-2006). Ziel ist dabei die Ermittlung des durchschnittlichen Lohnunterschieds zwischen Vollzeitbeschäftigten bei gleichen Berufen, gleichen beobachtbaren Eigenschaften der Arbeitskräfte und gleichen Eigenschaften der Betriebe. Dazu wird der Linked Employer-Employee-Datensatz des IAB (LIAB-Daten, vgl. Alda et al. 2005) herangezogen. In diesem Datensatz werden Individualdaten aus der Beschäftigtenhistorik des IAB für eine geschichtete Zufallsauswahl an Betrieben aufbereitet, die am IAB-Betriebspanel teilnehmen. Das IAB-Betriebspanel ist eine Befragung von Betrieben, die seit 1993 in Westdeutschland jährlich durchgeführt wird (vgl. Bellmann 2002). Die Individualdaten 
enthalten das Alter, das Geschlecht, den Bildungsabschluss sowie das sozialversicherungspflichtige Einkommen. ${ }^{2}$ Über die Betriebsnummer werden die Individualdaten mit den Betriebsdaten verknüpft. Ausgeschlossen werden Personen, die noch in der Ausbildung sind, und Personen mit unplausibel geringen Löhnen (weniger als das Doppelte der Geringfügigkeitsgrenze). Aufgrund des inhaltlichen Fokus auf eine bestmögliche Annäherung an eine etwaige unmittelbare Diskriminierung untersuchen wir vor allem die Lohnungleichheit innerhalb von Jobzellen (,within-job wage gap“). Solche Jobzellen setzen sich zusammen aus den Vollzeitbeschäftigten einer Berufsgruppe innerhalb eines Betriebes. Für die Definition der Berufsgruppe verwenden wir die amtliche Klassifikation der Bundesanstalt für Arbeit (Bundesanstalt für Arbeit 1992). Zur Einordnung der Ergebnisse stellen wir auch die Lohnunterschiede in den anderen Analyseebenen (Wirtschaftsbereiche, Berufe, Betriebe) und dem gesamten Arbeitsmarkt dar. Im Datensatz für 2006 finden sich 21.610 Jobzellen in 5.169 Betrieben, in 297 Berufsgruppen und aus 15 Wirtschaftsbereichen.

Zunächst geben wir zum Überblick an, wie hoch im gewichteten Durchschnitt die Löhne von Frauen in Prozent des Lohnes der Männer innerhalb der jeweiligen Analyseebene sind. Die Berechnung des relativen Lohnes der Frauen erfolgt nach:

$$
w_{r}=\frac{1}{N} \sum_{b=1}^{N} \frac{n_{b}}{n} \frac{\bar{w}_{b}^{f}}{\bar{w}_{b}^{m}} 100
$$

$\bar{w}_{b}^{f}$ ist der durchschnittliche Lohn der Frauen innerhalb des $b$-ten Sektors, Berufes, Betriebes bzw. der Jobzelle, $\bar{w}_{b}^{m}$ der entsprechende Lohn der Männer. Gebildet wird der gewichtete Mittelwert der $N$ Einheiten. Der Gewichtungsfaktor ist der Anteil der Beschäftigten in der jeweiligen Einheit $n_{b}$ an der Gesamtzahl der Beschäftigten $n$. Ein Wirtschaftsbereich, in dem eine geringe Anzahl von Beschäftigten arbeitet, geht damit mit einem geringeren Gewicht in den Mittelwert ein als ein Wirtschaftsbereich mit vielen Beschäftigten. $\mathrm{Zu}$ beachten ist, dass nur Einheiten mit mindestens einer Frau und mindestens einem Mann für Analysen herangezogen werden. Dies bedeutet, dass sich die ebenenspezifischen Auswertungen in den Fallzahlen unterscheiden.

Die ökonometrische Analyse folgt in theoretischer Hinsicht dem klassischen Humankapitalmodell (Mincer 1974). Die abhängige Variable ist der Tagesbruttolohn. Ein Problem des verwendeten Datensatzes besteht darin, dass die Einkommen der Beschäftigten nur bis zur Beitragsbemessungsgrenze gemeldet wurden. Personen, die über dieser Grenze verdienen, sind zwar im Datensatz vorhanden, statt des tatsächlichen Lohnes ist aber nur die Beitragsbemessungsgrenze angegeben. Die Daten sind also ab dieser Grenze zensiert.

2 Für den hier verwendeten Datensatz wurden alle Beschäftigungsmeldungen von Personen ausgewählt, die am 30. Juni des jeweiligen Jahres in einem Panelbetrieb gearbeitet haben. Da die Arbeitszeiten von Teilzeitbeschäftigten im Datensatz nur sehr grob erfasst sind, betrachten wir nur Vollzeitbeschäftigte. Systematisch ausgeschlossen sind im Datensatz Beamte, Selbstständige oder mithelfende Familienangehörige. Der Datensatz repräsentiert immerhin ca. 80\% der Erwerbstätigen. Eine weitergehende Beschreibung dieser Individualdaten findet sich in Bender et al. (1996). 
Um dadurch bedingte Verzerrungen der zu schätzenden Koeffizienten zu vermeiden, wurden die zensierten Werte durch Schätzungen ersetzt. ${ }^{3}$

Auf der Seite der unabhängigen Variablen nutzen wir den höchsten erworbenen Bildungsabschluss. Die Analysen von Hinz u. Gartner (2005) konnten die tatsächliche Berufserfahrung nicht einsetzen, da sie noch nicht im Datensatz erfasst war. Wir behalfen uns, wie bei Mincer-Schätzungen üblich (z. B Bellmann u. Möller 1995), mit der Konstruktion der potenziellen Berufserfahrung. Wir unterstellten somit für alle Beschäftigten ununterbrochene Erwerbsverläufe. Dies führt zu einer Überschätzung der Berufserfahrung von Frauen, bei denen häufiger Erwerbsunterbrechungen vorkommen als bei Männern. Inzwischen liegen individuelle Angaben der Erwerbsverläufe ab 1975 vor. Die Datenaufbereitung am IAB war überaus komplex. Dieser Fortschritt in der Datenaufbereitung ist für uns von zentralem inhaltlichen Interesse: Wie in Hypothese 1 formuliert, erwarten wir bei Verwendung der exakten Berufserfahrung in Jahren einen Rückgang des „within-job wage gap“.

Um dies zu untersuchen, führen wir Regressionsschätzungen mit fixen Effekten für die Ebene der Wirtschaftsbereiche, Berufe, Betriebe und Jobzellen durch (Greene 2000, S. $557 \mathrm{ff}$.). Wir illustrieren die Schätzgleichung für die Ebene der Jobzellen:

$$
\ln \left(w_{i j}\right)=\beta_{0}+\beta X_{i j}+\beta_{2} \text { frau }_{i j}+\varepsilon_{j}+\eta_{i j} .
$$

$\ln \left(w_{i j}\right)$ ist der logarithmierte Bruttotageslohn der Person $i$ in der Jobzelle $j . X_{\mathrm{ij}}$ ist ein Vektor mit den Humankapitalvariablen Ausbildung und Berufserfahrung ${ }^{4}, \eta_{i j}$ ein Störterm für die Personen. $\varepsilon_{\mathrm{j}}$ ist der fixe Effekt auf der Ebene der Jobzellen. Durch ihn werden Unterschiede $z w i s c h e n$ den Jobzellen aufgefangen. Die Variable frau ${ }_{\mathrm{ij}}$ nimmt den Wert eins an, wenn die Person eine Frau ist, ansonsten den Wert null. Der Koeffizient $\beta_{2}$ gibt nach Umrechnung mit $e^{\beta_{2}} \cdot 100$ den relativen Lohn von Frauen an. Mit $\left(e^{\beta_{2}}-1\right) \cdot 100$

3 Hierzu wurde der Lohn mit einem Tobit-Modell nach Geschlechtern getrennt geschätzt, wobei die in der Beschäftigtenstatistik zur Verfügung stehenden Variablen wie Branche und Humankapitalindikatoren als erklärende Faktoren verwendet wurden. Als Schätzer für den Lohn wurde der lineare Prädiktor X' verwendet, ergänzt um einen Störterm, der aus einer Zufallsverteilung mit der geschätzten Varianz des Störterms gezogen wurde. Dadurch wird verhindert, dass die geschätzten Löhne eine zu große Korrelation mit den erklärenden Variablen aufweisen. Da bekannt ist, dass der wahre Lohn über der Beitragsbemessungsgrenze liegt, wurde der Störterm aus einer gestutzten Verteilung gezogen, sodass der geschätzte Wert über dieser Schwelle liegt (siehe Gartner 2005).

4 Abhängige Variable: logarithmiertes Bruttotagesentgelt (mit imputierten Werten). Wir unterscheiden die Ausbildungskategorien: Ohne Ausbildung, Berufsausbildung, Abitur, Fachhochschule, Hochschule (Referenzkategorie: Berufsausbildung mit Abitur). Da die tatsächliche Berufserfahrung Unterbrechungen aufweist, wird sie anders modelliert als die potenzielle Berufserfahrung. Die tatsächliche Berufserfahrung wird abgebildet als Anteil der Beschäftigungsdauer in fünf Zeitintervallen (vor einem Jahr bis heute, vor vier bis vor einem Jahr, vor neun bis vier, vor 16 bis neun, länger als vor 16 Jahren). Die potenzielle Berufserfahrung wird linear, quadratisch und kubisch aufgenommen. Personen in Jobzellen, in denen nur Männer oder nur Frauen arbeiten, werden ausgeschlossen. Dies ist eine strengere Selektion als in Hinz u. Gartner (2005), die gewährleisten soll, dass die Ergebnisse der verschiedenen Analyseebenen besser verglichen werden können, da immer die gleiche Stichprobe verwendet wird. 
lässt sich berechnen, um wie viel Prozent der Lohn einer Frau geringer ist als der eines Mannes, wenn beide über ansonsten gleiche Eigenschaften verfügen.

Das Modell mit fixen Effekten ist als within-group-regression bekannt (Greene 2000, S. 562). Man erhält Schätzungen zum Lohnunterschied innerhalb der jeweiligen Einheiten (also Branchen, Berufe, Betriebe und Jobzellen). Modelle mit fixen Effekten benötigen vergleichsweise wenig restriktive Annahmen über die Verteilung der Fehlerterme. $\mathrm{Zu}$ beachten ist, dass keine ebenenspezifischen erklärenden Variablen in die Modelle aufgenommen werden können - etwa ob ein Betrieb einen Betriebsrat hat -, weil diese Eigenschaften für die Beschäftigten und Jobzellen ein und desselben Betriebs konstant sind. Für die Beschäftigten, die zur gleichen Analyseebene gehören, wird ein gemeinsamer fixer Effekt geschätzt, der die unbeobachtete Heterogenität der Analyseeinheiten modelliert.

\section{Ergebnisse}

Abbildung 1 gibt einen Überblick zur Entwicklung des relativen Lohns für den Gesamtarbeitsmarkt (Gesamt) und die vier Analyseebenen. $\mathrm{Zu}$ sehen sind von unten nach oben die relativen Löhne von Frauen im Gesamtarbeitsmarkt, innerhalb von Wirtschaftsbereichen (Branche), innerhalb von Berufen, innerhalb von Betrieben und innerhalb der Jobzellen. Die Entwicklung des relativen Lohns auf dem gesamten Arbeitsmarkt zeigt einen Rückgang der geschlechtsspezifischen Lohnlücke von 1993 bis 2000 von 24 auf weniger als $22 \%$. Danach vergrößert sich der durchschnittliche Lohnabstand wieder und liegt 2006 bei etwa $24 \%$. Damit entsprechen die Resultate ungefähr den von der EUKommission veröffentlichten Werten. ${ }^{5}$ Auch das leichte Anwachsen der Lohnlücke in jüngster Zeit, wie sie dort berichtet wird, ist in der Darstellung sichtbar. Die ebenenspezifischen Mittelwertberechnungen ergeben erwartungsgemäß jeweils höhere relative Löhne für Frauen und somit kleinere Lohnlücken. Für den Vergleich der Löhne in den Jobzellen, der niedrigsten Analyseebene, stellt man im gesamten Beobachtungszeitraum konstant einen relativen Lohn von Frauen zwischen 84 und 85\% fest. Innerhalb der niedrigeren Analyseebenen von Betrieben und Jobzellen stagnieren die Lohnunterschiede seit 1999. Halten wir also fest: Auf dem gesamten Arbeitsmarkt kann seit 1999 ein leicht wachsender Lohnabstand zwischen Frauen und Männern ausgemacht werden, was sich jedoch auf der Betriebsebene und in den Jobzellen nicht wiederfindet. $\mathrm{Zu}$ beachten ist, dass sich die Tendenz zu einer leicht wachsenden Lohnlücke aber bei Betrachtung der Wirtschaftsbereiche nachweisen lässt.

Ein entscheidender Fortschritt der vorliegenden Analyse gegenüber der Vorläuferarbeit besteht - wie herausgestellt - in der Berücksichtigung der Daten zur tatsächlichen Berufserfahrung, die einen wichtigen Bestimmungsfaktor für die Lohnhöhe darstellt. Wie vermutet, haben wir mit der konstruierten potenziellen Berufserfahrung für die vollzeitbeschäftigten Frauen die tatsächliche Berufserfahrung deutlich überschätzt (Medianver-

5 Die Werte der EU-Kommission beruhen allerdings auf Stundenlöhnen aller Beschäftigten in Deutschland. Unsere Werte beschränken sich auf sozialversicherungspflichtige Vollzeitbeschäftigte in Westdeutschland. 


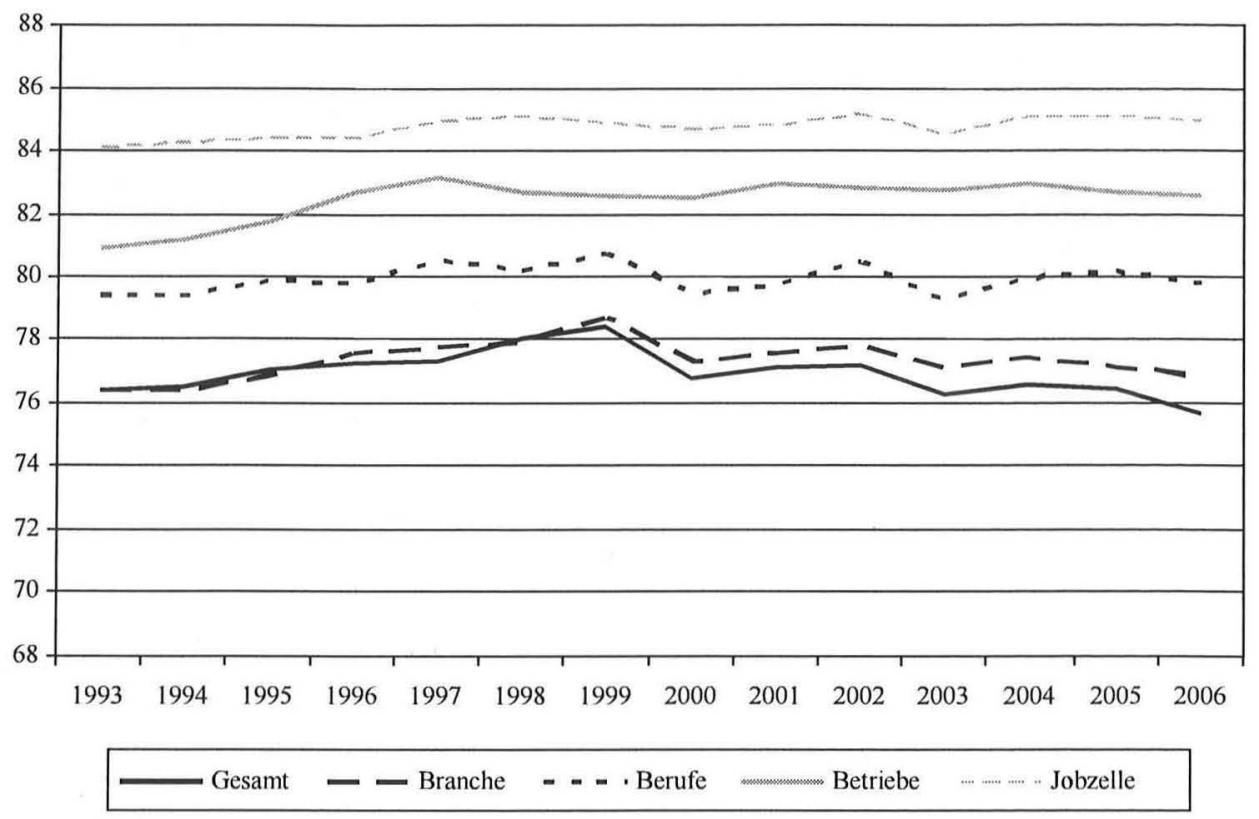

Quelle: LIAB, eigene Berechnungen

Abb. 1: Relativer Lohn von vollzeitbeschättigten Frauen (1993-2006), gewichtete Mittelwerte

gleich für 2006: 22,00 gegenüber tatsächlich 14,50 Jahren). Aber auch bei den Männern liegen die damals geschätzten (potenziellen) Werte über den tatsächlichen (Medianvergleich für 2006: 22,88 gegenüber tatsächlich 17,87 Jahren). Ein Vergleich der Verteilungen zeigt jedoch, dass hinsichtlich der Unterschiede zwischen den Geschlechtern beide Variablen für die Berufserfahrung insgesamt ein ähnliches Muster besitzen. ${ }^{6}$

Zur Untersuchung der Hypothese H 1, dass die unerklärten Lohnunterschiede bei genauerer Messung der erklärenden Variablen kleiner sind, betrachten wir die Schätzergebnisse des Modells mit fixen Effekten für 2006. Für die anderen Jahre fallen die Resultate ähnlich aus. In Abb. 2 sind die relativen Löhne von vollzeitbeschäftigten Frauen in Westdeutschland eingetragen, wobei für jede Analyseebene sowohl Schätzungen mit der potenziellen als auch mit der tatsächlichen Berufserfahrung durchgeführt wurden. Im Gesamtarbeitsmarkt beläuft sich der Lohn von Frauen relativ zum Lohn von Männern mit gleichem Humankapital auf einen Wert zwischen 83 und 84\%.

Damit beträgt der (zuvor ohne Humankapitalausstattung ermittelte, gewichtete mittlere) Lohnunterschied nicht mehr $24 \%$, sondern 16 bis $17 \%$. Der relative Lohn, der bei der Verwendung der tatsächlichen Berufserfahrung geschätzt wird, ist etwas höher als bei Verwendung der hypothetischen Werte für die Berufserfahrung. Dies entspricht den Überlegungen, die wir zu den Auswirkungen einer präziseren Erfassung der Berufserfahrung angestellt haben. Allerdings ist der Unterschied zwischen beiden Modellen nur gering

6 Die Verteilung der Variablen werden hier nicht eigens dargestellt. Auf Anforderung sind die Grafiken aber bei den Autoren erhältlich. 


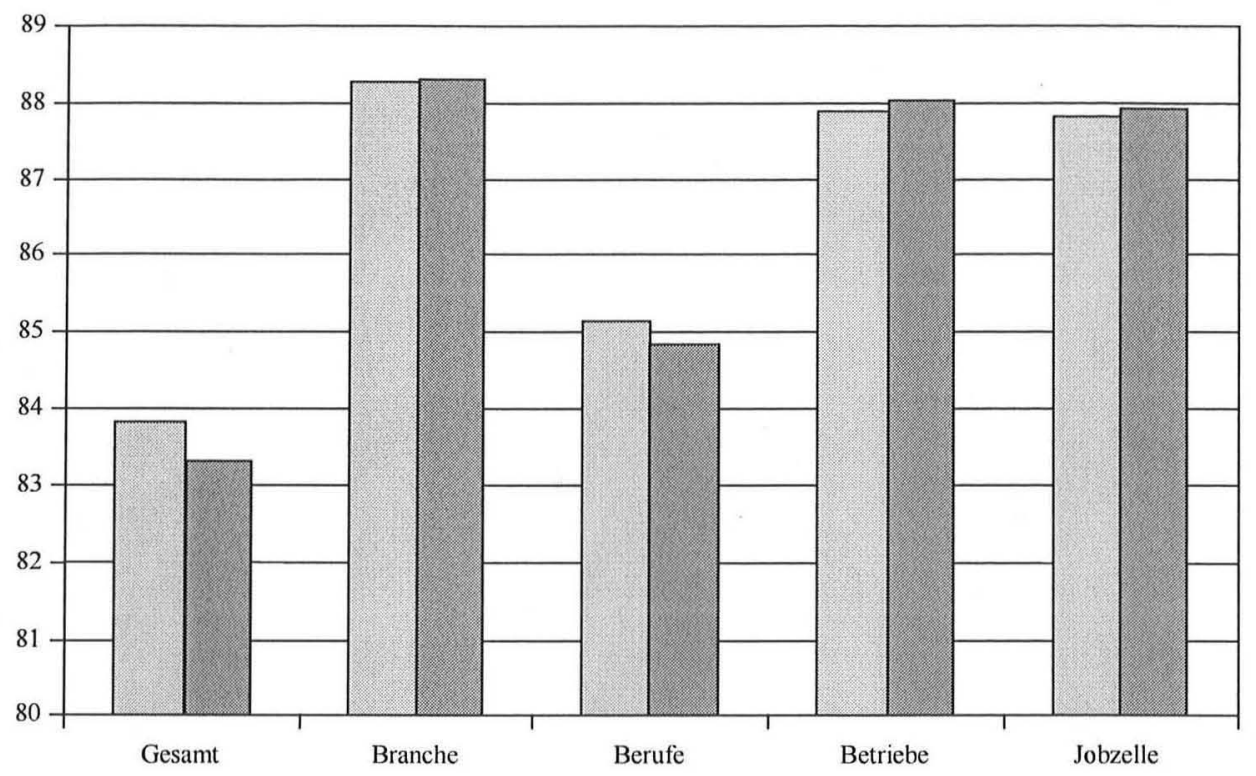

Tatsächliche Berufserfahrung $\square$ Potenzielle Berufserfahrung

Anmerkung: Abhängige Variable: logarithmierter Bruttotageslohn, unabhängige Variable: höchster Ausbildungsabschluss, tatsächliche Berufserfahrung als Anteil der Beschäftigungsdauer in fünf

Zeitintervallen (vor einem Jahr bis heute, vor vier bis einem Jahr, vor neun bis vier, vor 16 bis neun, länger als vor 16 Jahren), potenzielle Berufserfahrung linear, quadriert und kubisch, Quelle: LIAB, eigene Berechnungen

Abb. 2: Relativer Lohn von vollzeitbeschäftigten Frauen (2006), Schätzungen mit fixen Effekten und Humankapitalvariablen

$(0,5 \%)$. Abbildung 2 zeigt weiterhin: Der Lohnunterschied bei gleicher Humankapitalausstattung ist innerhalb von Wirtschaftsbereichen, innerhalb von Betrieben und innerhalb von Jobzellen deutlich geringer. Der relative Lohn von Frauen erreicht für diese Analyseebenen jeweils etwa $88 \%$. Für die Ebene der Berufe beträgt der relative Lohn von Frauen etwa $85 \%$. Zur Überprüfung von $\mathrm{H} 1$ ist das Modell für Jobzellen besonders einschlägig, weil wir uns damit dem Konzept einer möglichen unmittelbaren Diskriminierung am besten annähern können. Das Ergebnis der Schätzungen ist eindeutig: Die Lohnlücke geht bei Verwendung der rekonstruierten Berufserfahrung nicht weiter zurück, im Gegenteil, sie wird sogar minimal größer $(0,1 \%)$. Hypothese 1 muss somit als widerlegt gelten. Auch bei einer genaueren Messung der Berufserfahrung, die sich zwischen vollzeitbeschäftigten Frauen und Männern, wie berichtet, im Median um 3,37 Jahre unterscheidet, ist die Lohnlücke nicht geringer. Dies ist ein erstaunliches Ergebnis, welches in der Diskussion eingehender zu würdigen sein wird. Anzufügen ist, dass die Erklärungsleistung des Modells bei Verwendung der genaueren Messung dennoch besser ausfällt $\left(R^{2} 29,5\right.$ gegenüber 27,9).

Zur Überprüfung der Hypothese H 2, dass der „within-job wage gap“ zurückgegangen ist, ziehen wir ebenfalls die Ergebnisse der ökonometrischen Schätzungen heran, 


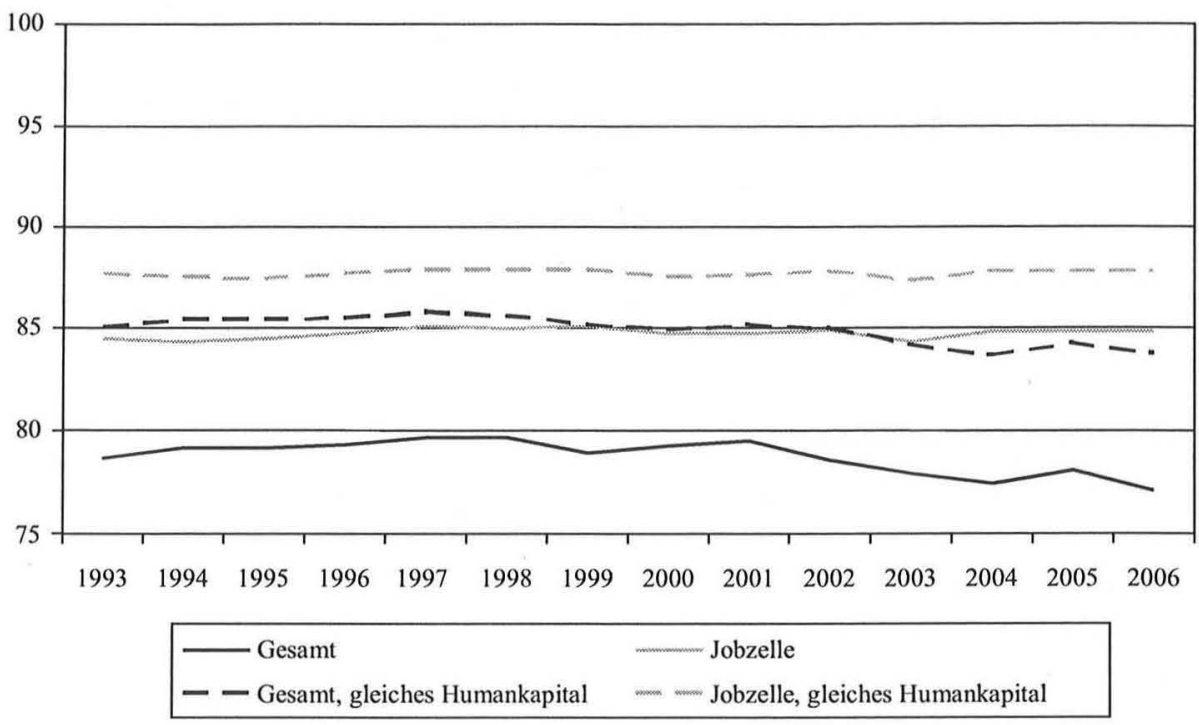

Anmerkung: abhängige Variable: logarithmierter Bruttotageslohn, unabhängige Variable: höchster Ausbildungsabschluss, tatsächliche Berufserfahrung (Modellierung siehe Abb. 2), Quelle: LIAB, eigene Berechnungen

Abb. 3: Relativer Lohn von vollzeitbeschäftigten Frauen (1993-2006), Schätzungen mit fixen Effekten

um mögliche Veränderungen in der Humankapitalausstattung zu berücksichtigen. Abbildung 3 zeigt zur besseren Vergleichbarkeit auch Schätzergebnisse für den Gesamtarbeitsmarkt und die Jobzellen ohne Einbezug von Humankapital. In etwa entsprechen die Schätzergebnisse den Resultaten für den Gesamtarbeitsmarkt (Gesamt) und die Jobzellen den Werten aus der Berechnung der gewichteten Mittelwerte (vgl. Abb. 1).

Wiederum erkennt man in jüngster Zeit ein geringfügiges Absinken des Lohns von Frauen gegenüber Männern, das sich jedoch nur im Gesamtarbeitsmarkt und nicht in den Jobzellen zeigt. Der Lohnunterschied ist erwartungsgemäß deutlich geringer, wenn Frauen und Männer mit gleicher Humankapitalausstattung verglichen werden. Beim Lohnunterschied in den Jobzellen ist allerdings kein klarer Trend ablesbar. Das Niveau der relativen Löhne von Frauen mit gleichem Berufsabschluss, gleicher Berufserfahrung im gleichen Betrieb und gleichem Beruf verharrt - anders als wir es nach $\mathrm{H} 2$ erwartet hätten - bei etwa $88 \%$. Dies ist ebenso erstaunlich, wenn man die lange Zeitreihe und die verschiedenen politischen und rechtlichen Maßnahmen gegen etwaige Lohndiskriminierungen in diesem Zeitraum bedenkt. Auch auf dieses Ergebnis kommen wir in der Diskussion zurück.

Zusätzlich zur Entwicklung der relativen Löhne ist es interessant zu untersuchen, wie sich die Erklärungsleistung der Schätzmodelle über die Zeit entwickelt hat. Die Erklärungskraft der Modelle mit der tatsächlichen und potenziellen Berufserfahrung ist seit 1993 rückläufig. In $\mathrm{Abb} .4$ sind die $\mathrm{R}^{2}$-Werte der within-Schätzungen für die Jobzellen eingetragen. Im Zeitverlauf verschlechtern sich die Schätzungen von $\mathrm{R}^{2}$-Werten von 0,35 auf 0,30 - wohlgemerkt bei Verwendung der gleichen erklärenden Variablen. Für die 


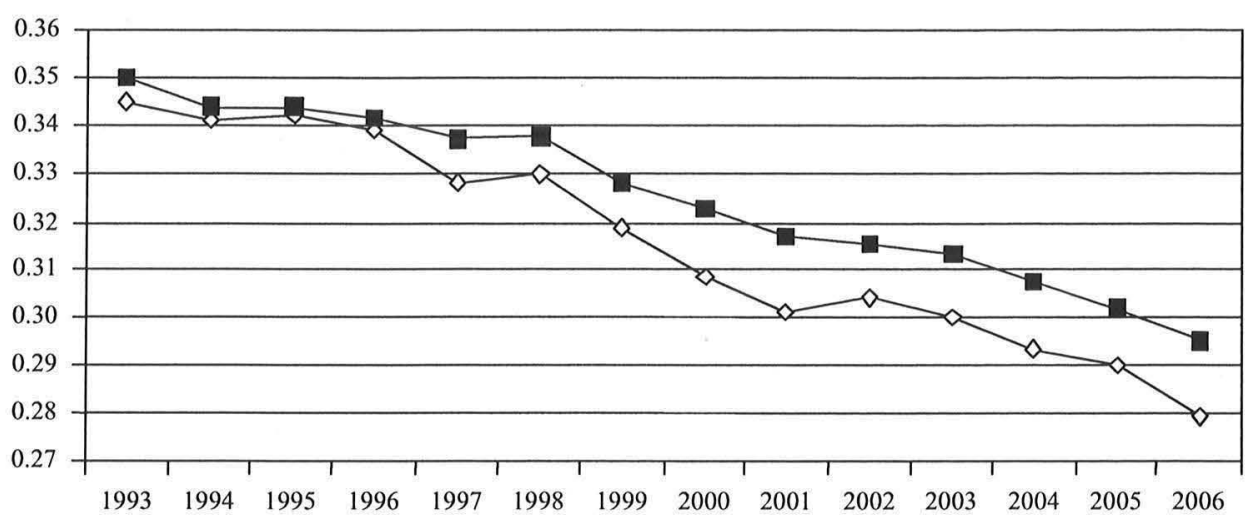

$\multimap$ within $\mathrm{R}^{2}$, potenzielle Berufserf. $\rightarrow$ within $\mathrm{R}^{2}$, tatsächliche Berufserf.

Anmerkung: Vergleich der Erklärungsleistung der Modelle mit der potenziellen und der tatsächlichen Berufserfahrung (Modellierung siehe Abb. 2), Quelle: LIAB, eigene Berechnungen

Abb. 4: Erklärungsleistungen der within-Jobzellen-Schätzungen des Bruttotageslohnes (erklärende Variablen: Humankapital und Geschlecht)

Untersuchung von Lohnunterschieden sind daher sowohl theoretische wie auch empirische Innovationen unabdingbar.

In theoretischer Hinsicht müssen die Mechanismen betrieblicher Lohnsetzung besser verstanden und in die Modelle integriert werden. Die Erklärungskraft der Modelle sinkt offenbar, weil die Lohnsetzung innerhalb von Betrieben und Jobzellen stärker differiert, was mit der größeren Flexibilität der Betriebe bei Lohnsetzungen zusammenhängen dürfte (vgl. auch Heinze u. Wolf 2006). In empirischer Hinsicht muss es gelingen, die „Künstlichkeit" der Jobkategorien, die auf einer veralteten Berufsklassifikation beruhen, zu überwinden. Die von uns angewandte Annäherung an den „within-job wage gap“ ist unter den gegebenen Bedingungen zwar ein Optimum. Angesichts einer sich deutlich verschlechternden Erklärungskraft des Modells darf sich die Arbeitsmarktforschung aber nicht auf die dauerhafte Anwendung eines offensichtlich immer schlechter der Realität entsprechenden Modells verlassen.

Der letzte Schritt der Auswertungen ist der Frage gewidmet, wie sich die Verteilung der Löhne von Frauen und Männern bis 2006 verändert hat. Bislang haben wir die Lohnlücke ausschließlich mit Hilfe der Mittelwerte beider Verteilungen betrachtet. Vor dem Hintergrund, dass die Lohnungleichheit in Deutschland mindestens seit Anfang der 2000er Jahre zunimmt (vgl. etwa Giesecke u. Verwiebe 2008), ist es erhellend zu sehen, wie zu drei Zeitpunkten (1993, 2000 und 2006) die jeweiligen Verteilungen aussehen. Die leichte Zunahme der Lohnungleichheit im Gesamtarbeitsmarkt kann schließlich mit geschlechtsspezifischen Veränderungen der Gesamtverteilung einhergehen.

In Abb. 5a ist zu erkennen: Frauen erhalten wesentlich häufiger Löhne im unteren Bereich der Lohnverteilung. Deren Verteilung ist auch weniger gespreizt als bei den Männern. Die Verteilung der Löhne von Männern weist nicht nur deutlich höhere Maße der 
Abb. 5a: Relative Häufigkeit der Bruttotageslöhne (2006) nach Geschlecht

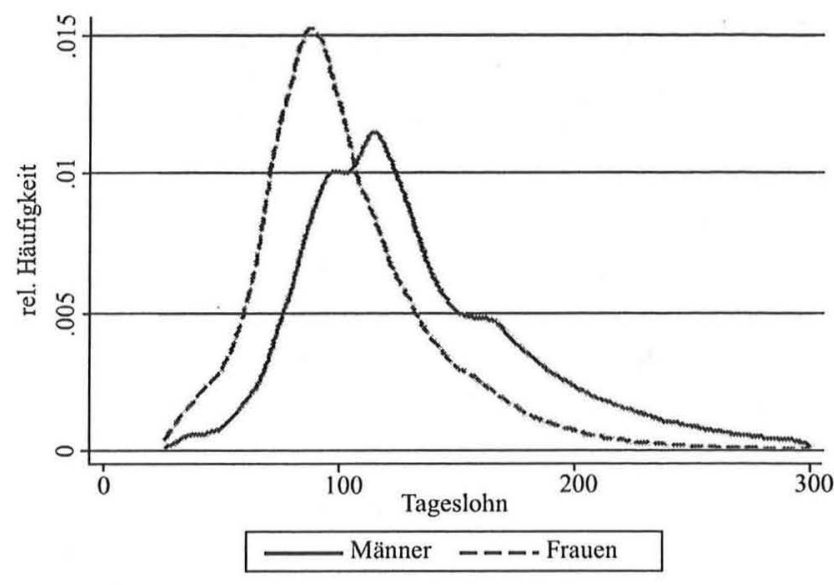

Quelle: LIAB, eigene Berechnungen

zentralen Tendenz auf, sie ist auch am rechten Ende (bei hohen Löhnen) „dicker“. Aber auch die Veränderungen im Zeitverlauf unterscheiden sich je nach Geschlecht, wenngleich nicht in der gleichen Deutlichkeit. Männliche Beschäftigte sind 2000 und 2006 noch häufiger als 1993 in oberen Verteilungssegmenten zu finden, während sich die Verteilung bei vollzeitbeschäftigten Frauen zwar auch nach oben verschoben, aber weniger deutlich verändert hat (vgl. Abb. 5b, c).

Zur Einschätzung der Veränderungen des durchschnittlichen Lohnes ist insbesondere dieser Trend einer stärkeren Spreizung der Männerlöhne von Bedeutung. Auch wenn sich in den Jobzellen nichts verändert (und sich die Indizien für unmittelbare Diskriminierungen zumindest nicht verschlimmern), kann der Lohnunterschied insgesamt zunehmen. Dies gilt insbesondere dann, wenn in den Hochlohnbereichen kaum noch Frauen und Männer in den gleichen Jobzellen miteinander verglichen werden können, weil es zu wenige Frauen in diesen Bereichen gibt.
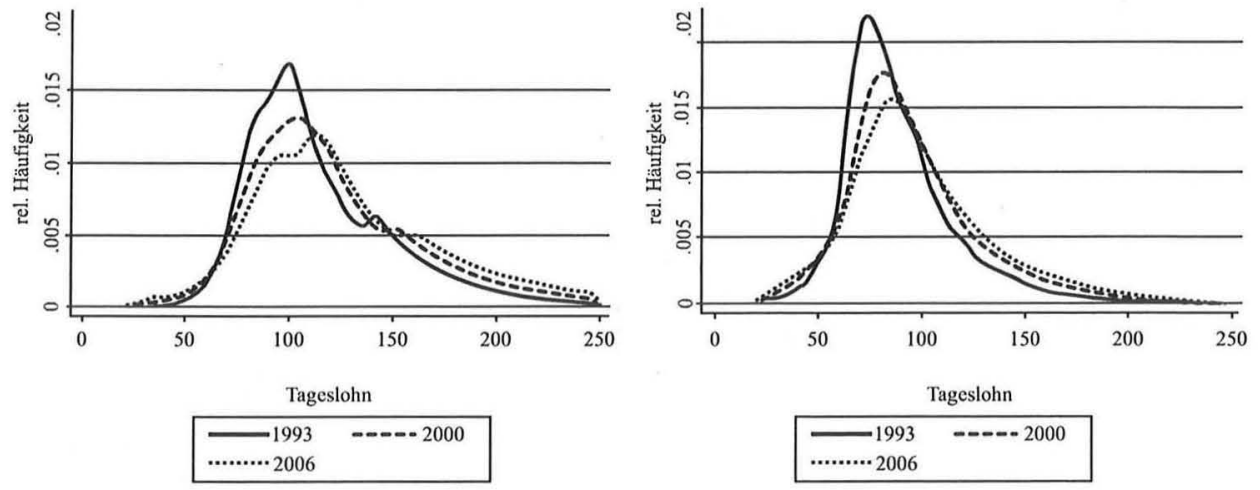

Männer

Frauen

Quelle: LIAB, eigene Berechnungen mit deflationierten Werten

Abb. 5b, c: Relative Häufigkeiten der Bruttotageslöhne (1993, 2000 und 2006) 


\section{Diskussion}

Die Frage nach Persistenz oder Wandel der geschlechtspezifischen Lohnungleichheit muss auf der Grundlage unserer Analysen eindeutig beantwortet werden: Am mittleren Lohnunterschied, den wir hier für vollzeitbeschäftigte Frauen und Männer in Westdeutschland ermittelt haben, hat sich in den letzten 15 Jahren nichts geändert, obgleich Politik und Öffentlichkeit den Lohnunterschied wesentlich deutlicher als früher als illegitim kennzeichnen. Sind die mehrfach verschärften gesetzlichen Bestimmungen - zuletzt im AGG von 2006 - nicht mehr als zahnlose Tiger?

Unsere Analysen, die eine gegenüber unserer eigenen Vorläuferstudie verbesserte Annäherung an den „within-job wage gap“ darstellen, zeigen, wie sehr die Antwort auf diese Frage immer noch von methodischen Unzulänglichkeiten im Datenbestand abhängt. Die im Datenbestand konstruierte Kategorie der Jobzelle dürfte in vielen Betrieben zu unscharf sein, insbesondere wenn es darum geht, hierarchische Strukturen innerhalb von Berufen zu erfassen. Fortschritte beim Abbau des illegitimen Lohnunterschieds sind an einen erhöhten Druck gegenüber den möglicherweise diskriminierenden Betrieben geknüpft. Dieser Druck kann aber nur entwickelt werden, wenn auch Instrumente für eine verbesserte Erfassung des „within-job wage gap“ zur Verfügung stehen. Die Bundesregierung prüft derzeit, ob das in der Schweiz implementierte Verfahren zur Bestimmung von betrieblicher Diskriminierung auch in Deutschland eingesetzt werden kann (Strub 2005). Dabei ist wesentlich, dass auch die hierarchischen Positionen von Beschäftigten und tatsächlichen Arbeitszeiten zuverlässig erfasst werden. Erst wenn die Messung diesbezüglich erheblich verbessert wird, werden die statistischen Analysen auf betrieblicher Ebene als mögliche Instrumente einer gerichtlichen Auseinandersetzung akzeptabel und wirklich belastbar. Der Abbau von möglicher Lohndiskriminierung setzt somit eine bessere Erfassung von Löhnen und Tätigkeiten voraus. Es müssen hierbei auch weitere betriebliche Variablen wie erfolgsabhängige Entlohnungssysteme, Arbeitszeitregelungen etc. beachtet werden. Unabhängig von der politischen und juristischen Auseinandersetzung über Diskriminierung müssen diese Informationen auch für die wissenschaftliche Bearbeitung erhoben werden. Dazu bedarf es - wie wir angesichts eines schleichenden Verfalls der Erklärungsleistung der Standardmodelle der Humankapitaltheorie anmerkten - auch theoretischer Anstrengungen.

Wie haben wir das zweite Hauptergebnis unserer Analysen zu deuten? Die präzise Messung von Berufserfahrung führt nicht zu einem statistischen „Wegerklären“ des Lohnunterschieds, sondern zu einer Erhöhung der statistischen Erklärungsleistung. Letzteres ist immerhin beruhigend. Eine methodische Antwort auf den ersten Teil des irritierenden Resultats lautet, dass bei Männern wie Frauen die potenzielle Verteilung der Berufserfahrung zwar verzerrt, aber in ähnlicher Weise verzerrt geschätzt wurde. In inhaltlicher Hinsicht ist wiederum die offensichtlich begrenzte Erklärungskraft des Mincer-Modells zu problematisieren. Während für den Gesamtarbeitsmarkt noch ein geringer Beitrag der genaueren Messung von Berufserfahrung zum Lohnunterschied nachweisbar ist, trifft dies für die Verhältnisse in den Jobzellen nicht zu. Möglich ist, dass auf der betrieblichen Ebene stärker als vermutet eine am schlichten Lebensalter orientierte Entlohnung praktiziert wird (vgl. schon Lazear 1981). Hinsichtlich interpersonaler Vergleiche spielt das Lebensalter eine wichtige Orientierungsfunktion und ist als Orientierungsrahmen prä- 
senter als die tatsächliche Berufserfahrung. Dies gilt wohl insbesondere für vollzeitbeschäftigte Personen. Bis bessere Erfassungsmethoden zur hierarchischen Besetzung von Positionen zur Verfügung stehen, können unsere Ergebnisse aber auch als Bestätigung eines im internationalen Vergleich weiterhin hohen Lohnunterschieds gelesen werden.

Neben aller Persistenz des Lohnunterschieds auf allen Ebenen ist auch auf offensichtliche Veränderungen hinzuweisen. Im letzten Analyseschritt konnte die Auswirkung einer sich verändernden Gesamtverteilung auf den geschlechtsspezifischen Lohnunterschied angedeutet werden. Die Lohnspreizung bei Männern ist größer geworden. Dies wirkt sich offensichtlich nicht auf den „within-job wage gap“ aus, weil Frauen in den Betrieben kaum oder nicht in den (neuen) Hoch- und Höchstlohnbereich vorstoßen. Weitergehende Analysen sollten prüfen, ob in den mittleren Bereichen der Verteilung bei Berücksichtigung von Eintrittskohorten nicht sogar ein Rückgang des Lohnunterschieds feststellbar ist. Damit ist eine sinnvolle Erweiterung der Analysestrategie angesprochen: Die Aufmerksamkeit sollte in Zukunft den Einstiegslöhnen sowie der individuellen Lohnentwicklung im Zeitverlauf gehören - immer unter Berücksichtigung der betrieblichen Kontexte. Die hier präsentierten Analysen können diese wichtigen Aspekte nicht aufgreifen. Ebenso muss auch die mögliche Veränderung der Selektion von vollzeitbeschäftigten Frauen und Männern in weiteren Untersuchungen beachtet werden. Auch ist zu berücksichtigen, dass der geschlechtsspezifische Lohnunterschied auf Veränderungen der Wirtschaftslage reagiert. Die aktuelle Wirtschaftskrise könnte bei erhöhter Arbeitslosigkeit den Lohnunterschied im Gesamtarbeitsmarkt verringern, während er in den Jobzellen konstant bleibt oder sogar zunimmt.

In diesem Artikel haben wir als beste Annäherung an die unmittelbare, evaluative Diskriminierung den „within-job wage gap“ herausgestellt. Damit der equal pay day in Zukunft näher an den 1. Januar rückt, muss breiter angesetzt werden. Besonders hartnäckig sind dem Arbeitsmarkt vorgeschaltete Prozesse wie die Entscheidung für Ausbildungsgänge, ob in der beruflichen Ausbildung oder im Studium. Hinzu kommen die kulturelle Entwertung von Tätigkeiten, die überwiegend von Frauen ausgeübt werden, und eine allokative Diskriminierung durch die Arbeitgeber. Die unmittelbare, zwar verbotene, aber schwer vor Gericht nachweisbare Diskriminierung dürfte - auch wenn sie in Deutschland ausgeprägter ist als in anderen Ländern - nur den kleineren Teil des Lohnunterschieds ausmachen.

\section{Literatur}

Achatz, J., Fuchs, S., Stebut, J. von, \& Wimbauer, C. (2002). Zur Beschäftigung hochqualifizierter Frauen. In J. Allmendinger \& T. Hinz (Hrsg.), Organisationssoziologie (S. 284-318). Sonderheft 42 der Kölner Zeitschrift für Soziologie und Sozialpsychologie. Opladen: Westdeutscher Verlag.

Achatz, J., Gartner, H., \& Glück, T. (2005). Bonus oder Bias? Mechanismen geschlechtsspezifischer Entlohnung. Kölner Zeitschrift für Soziologie und Sozialpsychologie, 57, 466-494.

Alda, H., Bender, S., \& Gartner, H. (2005). The linked employer-employee dataset created from the IAB establishment panel and the process-produced data of the IAB (LIAB). Schmollers Jahrbuch. Zeitschrift für Wirtschafts- und Sozialwissenschaften, 125, 327-336.

Altonji, J., \& Blank, R. (1999). Race and gender in the labor market. In Handbook of labor economics (Vol. 3C, Ch. 48, S. 3143-3259). Amsterdam: Elsevier. 
Arrow, K. J. (1974). Limited knowledge and economic analysis. American Economic Review, 64, $1-10$.

Baron, J. N., \& Newman, A. E. (1990). For what it's worth: Organizations, occupations and the value of work done by women and men. American Sociological Review, 55, 155-175.

Beblo, M., \& Wolf, E. (2003). Sind es die Erwerbsunterbrechungen? Ein Erklärungsbeitrag zum Lohnunterschied zwischen Frauen und Männern in Deutschland. Mitteilungen aus der Arbeitsmarkt- und Berufsforschung, 36, 560-572.

Becker, G. S. (1971). Economics of discrimination. Chicago: University of Chicago Press.

Bellmann, L. (2002). Das IAB-Betriebspanel: Konzeption und Anwendungsbereiche. Allgemeines Statistisches Archiv, 86, 177-188.

Bellmann, L., \& Möller, J. (1995). Institutional influences on interindustry wage differentials. In F. Buttler, W. Franz, R. Schettkat \& D. Soskice (Hrsg.), Institutional frameworks and labor market performance (S. 123-167). London: Routledge.

Bender, S., Hilzendegen, J., Rohwer, G., \& Rudolph, H. (1996). Die IAB-Beschäftigtenstichprobe 1975-1990. Beiträge zur Arbeitsmarkt- und Berufsforschung (Bd. 197). Nürnberg: Institut für Arbeitsmarkt- und Berufsforschung.

Berkovitch, N. (2001). Frauenrechte, Nationalstaat und Weltgesellschaft. In B. Heintz (Hrsg.), Geschlechtersoziologie (S. 375-379). Sonderheft 41 der Kölner Zeitschrift für Soziologie und Sozialpsychologie. Opladen: Westdeutscher Verlag.

Blau, F., Ferber, M., \& Winkler, A. (2006). The economics of women, men, and work. Upper Saddle River, NJ: Prentice-Hall.

Blau, F. D., \& Kahn, L. M. (1996). Wage structure and gender earnings differentials: An international comparison. Economica, 63, 29-62.

Bundesanstalt für Arbeit (1992). Schlüsselverzeichnis für die Angaben zur Tätigkeit in den Versicherungsnachweisen. Nürnberg: Internes Dokument der Bundesanstalt für Arbeit.

Darity, W., \& Mason, P. (1998). Evidence on discrimination in employment: Codes of color, codes of gender. Journal of Economic Perspectives, 12, 63-90.

Diekmann, A., Engelhardt, H., \& Hartmann, P. (1993). Einkommensungleichheit in der Bundesrepublik Deutschland: Diskriminierung von Frauen und Ausländern? Mitteilungen aus der Arbeitsmarkt- und Berufsforschung, 26, 386-398.

Fitzenberger, B., Schnabel, R., \& Wunderlich, G. (2004). The gender gap in labor market participation and employment: A cohort analysis for West Germany. Journal of Population Economics, $17,83-116$.

Gartner, H. (2005). The imputation of wages above the contribution limit with the German IAB employment sample. FDZ Methodenreport, 02/2005, Nürnberg.

Giesecke, J., \& Verwiebe, R. (2008). Die Zunahme der Lohnungleichheit in der Bundesrepublik. Aktuelle Befunde für den Zeitraum von 1998 bis 2005. Zeitschrift für Soziologie, 37, 403-422.

Greene, W. (2000). Econometric analysis. Upper Saddle River, NJ: Prentice-Hall.

Heckman, J. J. (1998). Detecting discrimination. Journal of Economic Perspectives, 12, 101-116.

Heinze, A., \& Wolf, E. (2006). Gender earnings gap in German firms: The impact of firm characteristics and institutions. ZEW Discussion Paper No. 06-020, Mannheim: ZEW.

Hinz, T., \& Gartner, H. (2005). Geschlechtsspezifische Lohnunterschiede in Branchen, Berufen und Betrieben. Zeitschrift für Soziologie, 34, 22-39.

Kommission der Europäischen Gemeinschaften (2007). Mitteilung der Kommission an das Europäische Parlament, den Europäischen Wirtschafts- und Sozialausschuss und den Ausschuss der Regionen - Bekämpfung des geschlechtsspezifischen Lohngefälles (18.07.2007). Brüssel KOM 2007/427.

Kommission der Europäischen Gemeinschaften (2009). Bericht zur Gleichstellung von Frau und Mann (27.02.2009). Brüssel KOM 2009/0077.

Lazear, E. P. (1981). Agency, earning-profiles, productivity and hours restriction. American Economic Review, 71, 606-620. 
Lazear, E. P. (1995). Personnel economics. Cambridge: MIT Press.

Lazear, E. P., \& Rosen, S. (1990). Male-female wage differentials in job ladders. Journal of Labor Economics, 8, 106-123.

le Grand, C., Szulkin, R., \& Tahlin, M. (1995). Why do some employers pay more than others? Earnings variation across establishments in Sweden. Research in Social Stratification and Mobility, 14, 265-296.

Lorenz, W. (1993). Diskriminierung. In B.-T. Ramb \& M. Tietzel (Hrsg.), Ökonomische Verhaltenstheorie (S. 119-144). München: Vahlen.

Mincer, J. (1974). Schooling, experience, and earnings. New York: Columbia University Press.

Nelson, R., \& Bridges, W. (1999). Gender inequality: Courts, markets, and unequal pay for women in America. Cambridge: Cambridge University Press.

Pager, D., \& Shephard, H. (2008). The sociology of discrimination: Racial discrimination in employment, housing, credit, and consumer markets. Annual Review of Sociology, 34, 181-209.

Petersen, T., Meyersson Milgrom, E. M., \& Snartland, V. (2003). The within-job gender wage gap, Sweden 1970-1990. Research in the Sociology of Organizations, 20, 319-353.

Petersen, T., \& Morgan, L. (1995). Separate and unequal: Occupation-establishment sex segregation and the gender wage gap. American Journal of Sociology, 101, 329-365.

Petersen, T., \& Saporta, I. (2004). The opportunity structure for discrimination. American Journal of Sociology, 109, 852-901.

Petersen, T., Snartland, V., Becken, L.-E., \& Olsen, K. M. (1997). Within-job wage discrimination and the gender wage gap: The case of Norway. European Sociological Review, 13, 199-213.

Phelps, E. S. (1972). The statistical theory of racism and sexism. American Economic Review, 62, 659-661.

Ramirez, F. O. (2001). Frauenrechte, Weltgesellschaft und die gesellschaftliche Integration von Frauen. In B. Heintz (Hrsg.), Geschlechtersoziologie (S. 356-374). Sonderheft 41 der Kölner Zeitschrift für Soziologie und Sozialpsychologie. Opladen: Westdeutscher Verlag.

Roos, P., \& Gatta, M. (1999). The gender gap in earnings. In G. N. Powell (Hrsg.), Handbook of gender and work (S. 95-123). Thousand Oaks: Sage.

Rupert, A., \& Voigt, M. (2009). Gehalt und Aufstieg. Mythen, Fakten, Modelle erfolgreichen Verhandelns. Aachen: Shaker Verlag.

Strub, S. (2005). Methodisches Vorgehen zur Überprüfung der Lohngleichheit zwischen Frau und Mann im Beschaffungswesen des Bundes. Bern: Eidgenössisches Büro für Gleichstellung.

Tam, T. (1997). Sex segregation and occupational gender inequality in the United States: Devaluation or specialized training? American Journal of Sociology, 103, 1652-1692.

Wharton, A. (2002). Geschlechterforschung und Organisationssoziologie. In J. Allmendinger \& T. Hinz (Hrsg.), Organisationssoziologie (S. 188-202). Sonderheft 42 der Kölner Zeitschrift für Soziologie und Sozialpsychologie. Opladen: Westdeutscher Verlag.

Wolf, E., \& Heinze, A. (2007). How to limit discrimination? Analyzing the effects of innovative workplace practices on intra-firm gender wage gaps using linked employer-employee data. ZEW Discussion Paper 07-077. Mannheim: ZEW.

Hermann Gartner, geb. 1968. Dr. oec. publ., Wissenschaftlicher Mitarbeiter am Institut für Arbeitsmarkt- und Berufsforschung, Nürnberg. Forschungsschwerpunkte: Lohnstrukturanalysen, Konjunktur und Arbeitsmarkt. Ausgewählte Veröffentlichungen: (mit H. Alda und S. Bender) The linked employer-employee dataset created from the IAB establishment panel and the process-produced data of the IAB (LIAB). In: Schmollers Jahrbuch. Zeitschrift für Wirtschafts- und Sozialwissenschaften 125, 2005; (mit S. Klinger) Ein janusköpfiger Aufschwung: Beschäftigungsgewinne und Polarisierung. In: WSI-Mitteilungen; 2008; (mit H. Alda/L. Bellmann) Wage structure and labor mobility in the private sector in West Germany 1993-2000. In: E. Lazear/K. Shaw (Hrsg.), An international comparison of the structure of wages, 2009. 
Thomas Hinz, geb. 1962. Prof. Dr. rer. pol., Professor für empirische Sozialforschung an der Universität Konstanz. Forschungsschwerpunkte: Methoden der empirischen Sozialforschung, Arbeitsmarktforschung, Wirtschafts- und Bildungssoziologie. Ausgewählte Veröffentlichungen: (mit Martin Abraham) Arbeitsmarktsoziologie, 2008; (mit Katrin Auspurg und Jürgen Güdler) Herausbildung einer akademischen Elite? Zum Einfluss der Größe und Reputation von Universitäten auf Forschungsförderung. In: Kölner Zeitschrift für Soziologie und Sozialpsychologie 60, 2008; (mit Thomas Wöhler) Egozentrierte Diskussionsnetzwerke in den USA und Deutschland. In: Sonderheft 47 der Kölner Zeitschrift für Soziologie und Sozialpsychologie, 2007. 